\title{
DME/TACAN Impact Analysis on GNSS Reflectometry
}

\author{
Raul Onrubia, Student Member, IEEE, Jorge Querol, Student Member, IEEE, Daniel Pascual, Student \\ Member, IEEE, Alberto Alonso-Arroyo, Student Member, IEEE, Hyuk Park, Senior Member, IEEE, \\ and Adriano Camps, Fellow, IEEE
}

\begin{abstract}
Global Navigation Satellite System Reflectometry (GNSS-R) is becoming a widely accepted technique for Remote Sensing. The interferometric technique (iGNSS-R) correlates the direct signal received from a satellite and the same signal reflected on the Earth's surface, whereas the conventional technique (cGNSS-R) correlates the reflected signal with a locally generated replica of the transmitted code. As GNSS signals are received below the noise level, this technique is extremely sensitive to Radio Frequency Interference (RFI). The Distance Measurement Equipment (DME), and the TACtical Air Navigation (TACAN) systems are two radio navigation systems that transmit in the GPS L5, and Galileo E5 bands with powers up to $3.5 \mathrm{~kW}$. This work studies in depth the impact of these systems on iGNSS$R$, and cGNSS-R instruments. This study is then applied to an hypothetical reflectometer that will be placed in the International Space Station (ISS): the GEROS experiment. It is shown that the received power in space will be strong enough to degrade the system's performance by increasing the noise floor, but the sea altimetry precision will still be accurate enough for scientific studies.
\end{abstract}

Index Terms-GNSS-R, Interferometric, Radio Frequency Interference, DME, TACAN, RFI

\section{INTRODUCTION}

G LOBAL Navigation Satellite Signals (GNSS) are becoming popular as signals of opportunity for reflectometry (GNSS-R) since 1988 when it was first proposed for scatterometry [1], and later on, in 1993 for multistatic mesoscale altimetry [2]. Therein, the so-called Interferometric technique (iGNSS-R) was proposed, which is based on the cross-correlation in the delay $(\tau)$ and Doppler frequency $(\nu)$ domains (Delay-Doppler Map or DDM) between the direct transmitted signal from the satellite $y_{d}$, and the same signal reflected on the Earth's surface $y_{r}$ [3]:

$\left\langle\left|Y_{y_{d} y_{r}}(\tau, \nu)\right|^{2}\right\rangle=\left\langle\left|\frac{1}{T_{c}} \int_{0}^{T c} y_{d}(t) y_{r}^{*}(t-\tau) e^{-j 2 \pi \nu t} d t\right|^{2}\right\rangle$,

where $T_{c}$ is the coherent integration time. Equation (1) can be understood as the output power after correlating the signal $y_{r}$ with a matched filter with an impulse response defined by $y_{d}$

This work has received funding from the European Union's Seventh Framework Programme for research, technological development and demonstration under grant agreement "European GNSS-R Environmental Monitoring" $\mathrm{n}^{\mathrm{O}}$ FP7-607126-E-GEM, from the project ESP2015-70014-C2-1-R of the MINECO, and from the Phase A study of the ESA project "GNSS Reflectometry, Radio Occultation and Scatterometry on-board ISS (GEROSISS)". for different delays and Doppler frequencies. Therefore, the signal $y_{d}$ is treated as unit-less.

Later on, in 1996, it was proposed by [4] the conventional technique (cGNSS-R), which consists on the correlation of the reflected signal over the Earth's surface $y_{r}$ against a locally generated clean replica of the code $c$, achieving in this way higher Signal-to-Noise Ratio (SNR). However, this technique can only be used with open codes, which have narrow bandwidths (typically $2 \mathrm{MHz}$, but up to $20 \mathrm{MHz}$ ), and therefore, low precision. On the opposite side, iGNSS-R can cross-correlate any signal (even the military M-code, with 30 $\mathrm{MHz}$ of bandwidth), achieving higher precision at the expense of a reduction of the SNR.

Except GLONASS, all GNSS use Code Division Multiple Access (CDMA) to multiplex the signals transmitted by different satellites, while at the same time it achieves a large correlation gain after despreading the received signal. Despite this, as GNSS signals are received below the noise floor they are vulnerable to any Radio Frequency Interference (RFI). This might become an important problem in GNSS-R, and particularly in iGNSS-R, considering that some GNSS bands are shared with other services. In particular, the Global Positioning System (GPS) L5 band (1,164 - 1,188 MHz), the Galileo E5 band (1,166 - 1,217 MHz), and the BeiDou B2 band (1,194 - 1,219 MHz) coexist with two radio navigation systems: a civilian one called Distance Measurement Equipment (DME), and a military one called TACtical Air Navigation system (TACAN). Both systems transmit in the band from $962 \mathrm{MHz}$ to $1,213 \mathrm{MHz}$ with powers reaching up to $3.5 \mathrm{~kW}$ [5].

The impact of radio navigation signals on GNSS navigation, and the evaluation of several mitigation techniques have been widely studied. The impact of several airport equipments in a Galileo receiver has been studied in [6], and concluded that "DME signals are the most significant interference" and that a mitigation technique was needed to avoid the receiver to loose tracking. It has been predicted a degradation up to $12 \mathrm{~dB}$ over Europe at 12200 meters height in [7], and proposed a pulse blanking system to overcome this problem. Navigation systems cross-correlate the direct signal $y_{d}=d(t)+i_{d}(t)+n_{d}(t)$, and a clean replica of the code $c(t)$ [8]:

$$
Y_{y_{d} c}(\tau, \nu)=Y_{d c}(\tau, \nu)+Y_{i_{d} c}(\tau, \nu)+Y_{n_{d} c}(\tau, \nu)
$$

where $d$ is the clean GNSS received signal, $i$ is the interference signal, $n$ is noise, and $c$ is the code used to despread the GNSS signal $d$. The used codes are the so-called Pseudo-Random 
Noise (PRN) sequences. These codes are robust against interferences, and noise [9] because in the cross-correlation process the power spectrum of the interference signal is spread, and the spectral density of the interference over the GNSS resulting band decreases. Even with this spreading effect, if the interference is powerful enough, the terms $Y_{i c}$ and $Y_{n c}$ can reduce the signal quality, even to critical levels making impossible the use of GNSS [10]. Analogously, in the case of cGNSS-R the code $c(t)$ is correlated with the reflected signal $y_{r}=r(t)+i_{r}(t)+n_{r}(t)$ :

$$
Y_{c y_{r}}(\tau, \nu)=Y_{c r}(\tau, \nu)+Y_{c i_{r}}(\tau, \nu)+Y_{c n_{r}}(\tau, \nu) \text {. }
$$

Since the reflected signal is even weaker than the direct one, it is expected that in GNSS-R the degradation to be much worse than in GNSS navigation. In navigation the antenna is pointing towards the sky, so a receiver located in an airplane receives the DME signals attenuated from the back lobes of the antenna. However, in reflectometry the antenna is pointing towards the ground, so the interference is stronger as it is received from the main beam of the antenna. Besides, the GNSS signal is received approximately $25 \mathrm{~dB}$ weaker due to the scattering process in the Earth surface [11].

In the iGNSS-R technique the direct signal $y_{d}$ is crosscorrelated against the reflected signal $y_{r}$ :

$$
\begin{aligned}
Y_{y_{d} y_{r}}(\tau, \nu)= & Y_{d r}(\tau, \nu)+Y_{d i_{r}}(\tau, \nu)+Y_{d n_{r}}(\tau, \nu) \\
& +Y_{i_{d} r}(\tau, \nu)+Y_{i_{d} i_{r}}(\tau, \nu)+Y_{i_{d} n_{r}}(\tau, \nu) \\
& +Y_{n_{d} r}(\tau, \nu)+Y_{n_{d} i_{r}}(\tau, \nu)+Y_{n_{d} n_{r}}(\tau, \nu),
\end{aligned}
$$

where $r$ is the clean reflected GNSS received signal, $i_{d}$ and $i_{r}$ are the interferences received by the direct and reflected antennas respectively, and $n_{d}$ and $n_{r}$ are the noise terms in the direct and reflected signals respectively. $Y_{d r}$ is the useful part of the DDM. In the DME case, $Y_{i_{d} i_{r}}$ is expected to be the dominant term of the undesirable terms due to the high transmitted power. The other terms both for cGNSS-R and iGNSS-R were studied in [12], but not particularizing for any kind of interfering signal.

This work is devoted to study the impact of the DME/TACAN signals in cGNSS-R, and in iGNSS-R. First, the DME/TACAN signals and their correlation properties are studied in Section II. Then, Section III shows the computation of the DME/TACAN power that would reach a low earth orbiter, in particular the GEROS-ISS, an interferometric reflectometer that will be placed in the International Space Station (ISS) [13]. Section IV shows and discusses the resulting global maps, and evaluates the weight of each interfering term in (3) and (4). Finally, the main conclusions are summarized in Section V.

\section{DME/TACAN SIGNALS}

GPS L5, and the Galileo E5 bands (1,164 MHz - 1,217 $\mathrm{MHz}$ ) are shared with two wide extended aerial radio navigation systems: the DME (civilian), and TACAN (military), both transmitting in the band from $962 \mathrm{MHz}$ to $1,213 \mathrm{MHz}$, divided in $1 \mathrm{MHz}$ channels [5]. Both systems are based on the time delay to determine the distance between an aircraft, and a transponder. When a plane interrogates a station, it transmits a sequence of pairs of pulses with an average pulse repetition frequency of 27 pulses per second at the frequency channel assigned to the transponder. When a pair of pulses reaches the DME/TACAN station, it is retransmitted at a different frequency channel after a given delay, depending on the channel and the coding assigned to each transponder. The distance to the station is then estimated from the elapsed time between the transmission and the reception of the pulse $t_{0}$, the plane height, and the delay at the station $t_{D}$, as illustrated in Fig. 1.

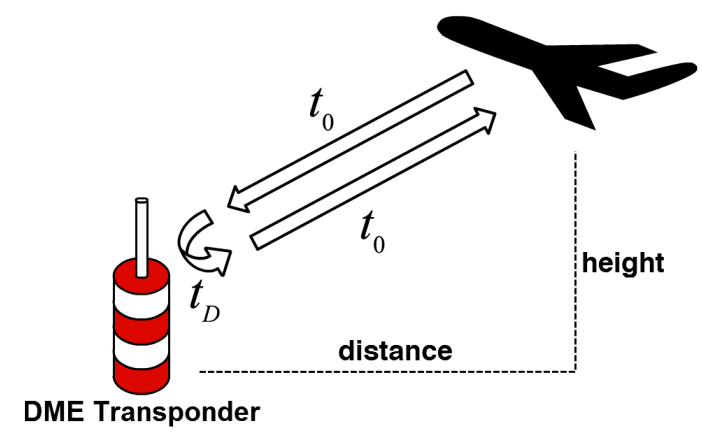

Fig. 1: Simplified DME concept.

DME signals (see Fig. 2) consist of pairs of Gaussian pulses of $12 \mu \mathrm{s}$ length with the following expression:

$$
s(t)=\left(e^{-\frac{\alpha}{2}\left(t-\frac{\Delta t}{2}\right)^{2}}+e^{-\frac{\alpha}{2}\left(t+\frac{\Delta t}{2}\right)^{2}}\right) \cdot \cos \left(2 \pi f_{c} t+\phi\right)
$$

where $\alpha=4.5 \cdot 10^{11} \mathrm{~s}^{-2}, f_{c}$ is the channel frequency, and $\Delta t$ is the time separation between pulses.

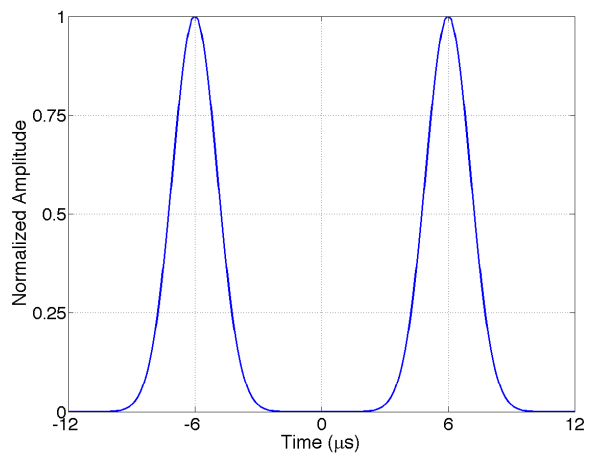

Fig. 2: DME pulse shape.

The separation is determined by the operation mode, and the coding. The operation modes, DME/N and DME/P, are used to provide different levels of accuracy appropriate for each flying operation. DME/N is used for on route navigation, and has an accuracy of 370 meters. DME/P is used for precise operations such as airport approaching (Initial Approach or DME/P IA, with an accuracy from 370 to 85 meters), and landing (Final Approach, or DME/P FA, with an accuracy down to 12 meters). The codes, $\mathrm{X}$ and $\mathrm{Y}$, allow to reuse each frequency channel more than once. DME transponders have an 
TABLE I: DME transmitting and receiving frequencies (from the airplane point of view).

\begin{tabular}{|l|r|r|r|}
\hline Coding & Channel & $\begin{array}{r}\text { Transmitting } \\
\text { Frequency }(\mathrm{MHz})\end{array}$ & $\begin{array}{r}\text { Receiving } \\
\text { Frequency }(\mathrm{MHz})\end{array}$ \\
\hline $\mathrm{X}$ & $1 \rightarrow 63$ & $1,025 \rightarrow 1,087$ & $962 \rightarrow 1,024$ \\
& $64 \rightarrow 126$ & $1,088 \rightarrow 1,150$ & $1,151 \rightarrow 1,213$ \\
\hline $\mathrm{Y}$ & $1 \rightarrow 63$ & $1,025 \rightarrow 1,087$ & $1,088 \rightarrow 1,150$ \\
& $64 \rightarrow 126$ & $1,088 \rightarrow 1,150$ & $1,025 \rightarrow 1,087$ \\
\hline
\end{tabular}

assigned channel from among the 126 existing, and a coding. The assigned transmitting and receiving frequencies are fixed and can be seen in Table I. Stations using the $X$ coding and channels from 77 to 126 retransmit the received signals at frequencies from 1,164 MHz to $1,213 \mathrm{MHz}$, where the $\mathrm{L} 5$ and E5 bands are allocated. The time separation between pulses for the interfering transponders is $\Delta t=12 \mu \mathrm{s}$ [5]. Airplanes transmit out of the GNSS band (1,025 - 1,150 MHz), as well as stations with Y coding.

According to $[14]^{1}$, from a total of 4,000 DME and TACAN stations, more than 2,500 are assigned to the $77-126$ channels in X mode (see Fig. 3).

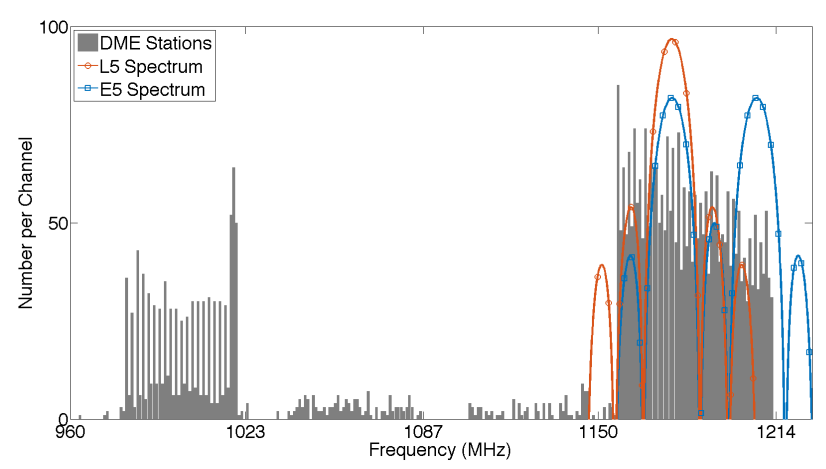

Fig. 3: DME channel allocation (grey), L5 spectrum (orange) and E5 spectrum (blue).

As it can be seen in Fig. 4, North America, Europe, and the East Asia are the regions with the highest density of DME/TACAN stations; therefore these regions are specially susceptible to RFI.

\section{A. Signal properties}

DME signals retransmitted from the transponder reach the up- and down-looking antennas of the reflectometer with a negligible time difference (as compared to the sampling frequency), with the same Doppler frequency, but with different phase and amplitude due to the separation between antennas and their orientation. Therefore, in interferometric reflectometry, if the direct and the reflected signals are sampled simultaneously ("non-delayed cross-correlation"), and the correlation peak in the DDM is always centered at the origin. Figure 5 shows the Woodward Ambiguity Function (WAF) [15] of

\footnotetext{
${ }^{1}$ There are no official public world databases of Radio Navigation Aids since the Digital Aeronautical Flight Information File (DAFIF) was closed in 2006. However, several unofficial databases have appeared, such as OurAirports, which mixes multiple official regional sources in a single database.
}

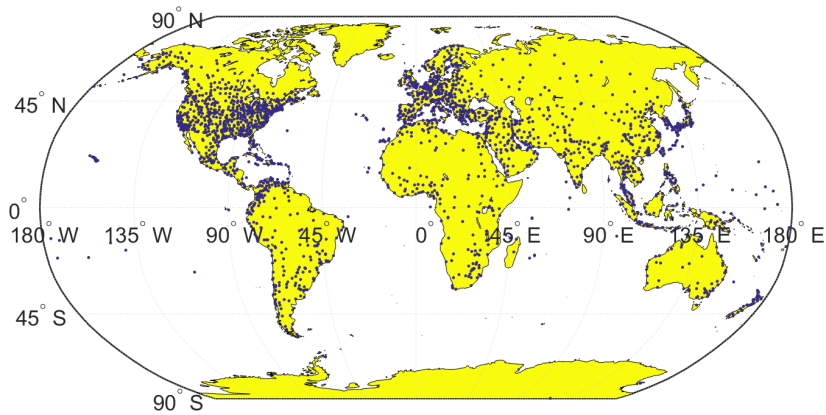

Fig. 4: DME station's locations [14].

a single DME pair of pulses, which is the auto-correlation function in the delay and Doppler domain $Y_{s s}(\tau, \nu)$. As it can be seen, in the Doppler domain the main lobe is modulated by a $1 /(12 \mu s) \approx 83 \mathrm{kHz}$ frequency, with a bandwidth of $\approx 40$ $\mathrm{kHz}$ for the central peak.

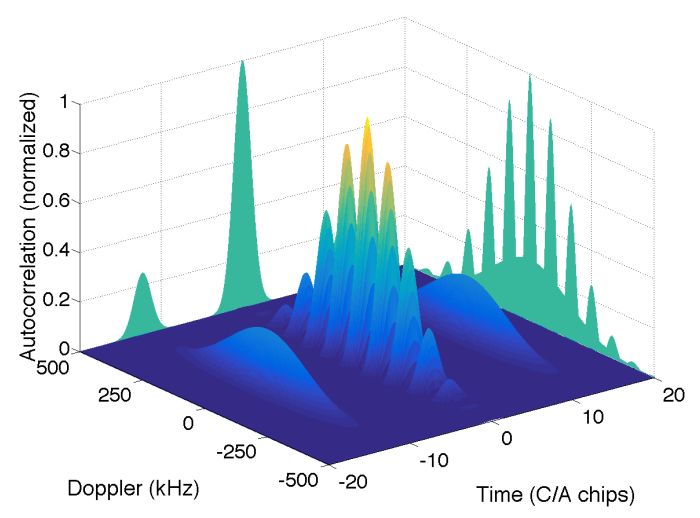

Fig. 5: DME Woodward Ambiguity Function (WAF).

When $N$ signals from different transceivers are received at the direct and reflected antennas, $N$ cross-correlation peaks appear at the origin of the DDM. All these signals also correlate at 0-delay for multiple Doppler frequencies, but not as strongly. The signals received from two different stations have different delays and Doppler frequencies; and therefore, $N^{2}-N$ cross-correlation peaks appear spread in the DDM plane. When several correlations are incoherently averaged, the contributions at 0-delay are always present, but the other ones spread along the DDM, and they are not in the same position. Therefore, the energy of the DDM tends to concentrate along the 0-delay as well (Fig. 6b). If the number of DME signals present is small, the resulting DDM is almost constant for any Doppler frequency as it can be appreciated in Fig. 6a. However, as the number of DME pulses increases, the WAF changes and the energy of the DDM tends to concentrate also around the 0-Doppler. Figure 6 shows the resulting WAF after the incoherent averaging of 50,000 simulated WAFS with (a) 1 pair of pulses present in the coherent integration time, and (b) 10 pairs of pulses for the "non-delayed cross-correlation" considering uniformly distributed random Delay (as will be seen later in section II-B) and Doppler between pulses. 


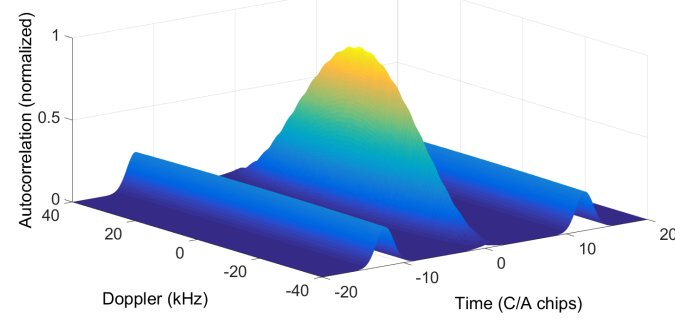

(a)

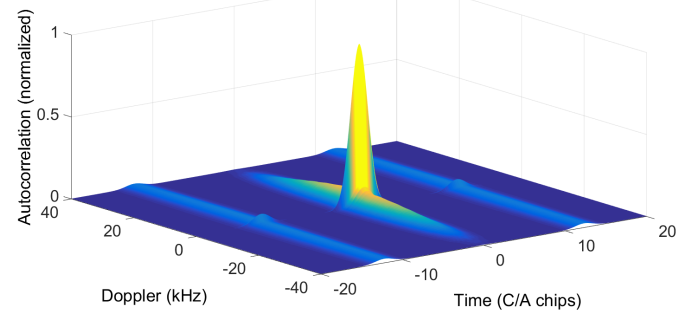

(b)

Fig. 6: Resulting WAF for "non-delayed cross-correlation" after the incoherent averaging of 50,000 simulated WAFS considering uniformly distributed random Delay and Doppler between pulses with (a) 1 pair of pulses present in the coherent integration time, and (b) 10 pairs of pulses.

The higher the altitude of the GNSS reflectometer, the longer the delay of the reflected signal as compared to the the direct signal, so the latter has to be delayed prior to the correlation [16] ("delayed cross-correlation"). Therefore, the sequences of pulses in the direct and reflected signals are different, so they do not cross-correlate at the origin of the DDM, but are uniformly distributed over the delay axis. The Doppler frequency difference between pulses from a same DME station is almost zero as it changes less than $1 \mathrm{~Hz} / \mathrm{ms}$ [17], so the cross-correlation peaks between same stations are uniformly distributed along the $0 \mathrm{~Hz}$ Doppler line. Moreover, the Doppler frequency and delay between different stations is random, so it is the position of these cross-correlation peaks in the DDM plane. As a consequence, the WAF is uniform in delay, and it has a bit more energy for lower Doppler frequencies as it can be seen in Fig. 7. Increasing the number of pulses does not affect noticeably the shape of the DDM, except for its level that is increased, but it cannot be appreciated due to amplitude normalization. This figure shows the resulting WAF after the incoherent averaging of 50,000 simulated WAFS with (a) 1 pair of pulses present in the coherent integration time, and (b) 10 pairs of pulses for the "delayed cross-correlation" considering uniformly distributed Delay and Doppler frequency between pulses.

The DME signals from different stations cross-correlate between them in the DDM plane only if they are allocated in the same frequency channel, i.e. the cross-correlation between signal in different channels will not be considered. The DME spectrum is $1 \mathrm{MHz}$ wide, and the channels are separated by 1

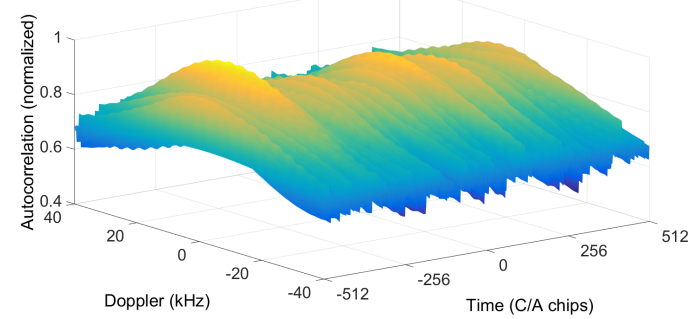

(a)

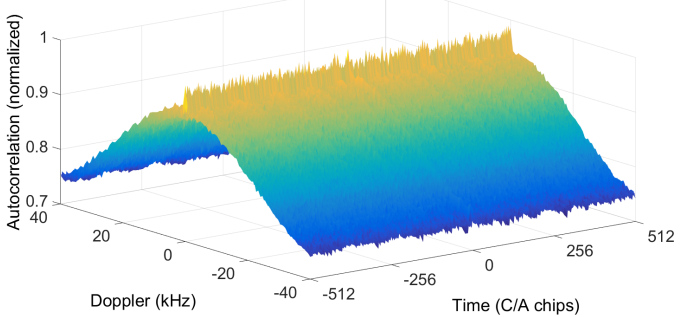

(b)

Fig. 7: Resulting WAF for "delayed cross-correlation" after the incoherent averaging of 50,000 simulated WAFS considering uniformly distributed Delay and Doppler between pulses with (a) 1 pair of pulses present in the coherent integration time, and (b) 10 pairs of pulses.

MHz. The maximum Doppler of a received DME signal was determined by simulation to be lower than $\pm 28 \mathrm{kHz}$ in the spaceborne case. To do so, AGI STK was used to compute the received Doppler shift in a LEO orbit from several DME stations at different locations. The expected Doppler difference between the received GNSS signals in the up and downlooking antennas will no exceed $\pm 40 \mathrm{kHz}$ [17], so the DDM does not have be computed out of this range. As consequence, the part of the spectrum of two DME signals in adjacent channels that could overlap has a negligible amount of power.

From (4), the average power in $1 \mathrm{~ms}$ after correlating two DME signals $Y_{i i}$ in the "delayed cross-correlation" can be estimated as:

$\left\langle\left|Y_{i_{d} i_{r}}(\nu)\right|^{2}\right\rangle=\sum_{k} \sum_{l} \bar{P}_{i_{d_{k}}} \cdot \bar{P}_{i_{r_{l}}} \cdot \delta\left(f_{k}-f_{l}\right) \cdot \eta_{A C F} \cdot \Upsilon(\nu)$,

where $\bar{P}_{i_{d, r}}$ is the average power ${ }^{2}$ of the DME signal in $1 \mathrm{~ms}$, $f$ is the frequency channel at which is transmitted, $\delta\left(f_{k}-f_{l}\right)$ is used to only compute the power of signals allocated in the same frequency channel, $\eta_{A C F}=-24,1 \mathrm{~dB}$ is the average value along the delay axis of an ACF of an unitary mean power DME signal, and $\Upsilon(\nu)$ accounts for the effect of the spreading of the DME WAF in the DDM plane due to the relative Doppler between stations. $\Upsilon(\nu)$ is calculated statistically depending on the maximum Doppler between stations and its probability distribution. $\bar{P}_{i_{d, r}}$ is calculated with the DME peak power

\footnotetext{
${ }^{2}$ Recall that, following the definition in (1), $y_{D}$ is unitless and therefore, $P_{i_{d}}$ is unitless as well, while $P_{i_{r}}$ has units of Watts.
} 
$P_{i_{d, r}}$, the average number of received pulses per ms $\bar{N}$, and the scaling factor between the DME peak power and the DME average power in $1 \mathrm{~ms} \eta_{D M E}=-24,3 \mathrm{~dB}$ as:

$$
\bar{P}=\left(P \cdot \eta_{D M E} \cdot \bar{N}\right)
$$

As aforementioned, in the "non-delayed cross-correlation" case the cross-correlation peaks between the same station are concentrated in the 0-Delay and 0-Doppler position. Therefore, to compute the term $Y_{i_{d} i_{r}}$ at 0 second Delay and $0 \mathrm{~Hz}$ Doppler, equation (6) is evaluated with $\eta_{A C F}=1$ for the terms that fulfill $k=l$, whereas the other points are computed evaluating the equation only for $k \neq l$.

The rest of the noise and interfering cross-terms $Y_{d i_{r}}, Y_{d n_{r}}$, $Y_{i_{d} r}, Y_{i_{d} n_{r}}, Y_{n_{d} r}, Y_{n_{d} i_{r}}$, and $Y_{n_{d} n_{r}}$ are estimated in a general form as:

$$
\left\langle\left|Y_{s_{1} s_{2}}\right|^{2}\right\rangle=P_{s_{1}} \cdot P_{s_{2}} \cdot \gamma_{s_{1} s_{2}}
$$

where $s_{1}$ and $s_{2}$ are the direct GNSS clean signal $d$, the reflected GNSS clean signal $r$, the interference signal received in the up-looking and the down-looking antennas $i_{d}$ and $i_{r}$ respectively, or the noise signals in the direct and reflected antenna $n_{d}$ and $n_{r}$ respectively. $P$ is the average power of the signal in $1 \mathrm{~ms}$, which in the case of DME signals is calculated in (7).

$\gamma_{s_{1} s_{2}}$ is the Generalized Spectral Separation Coefficient (GSSC) between signals $s_{1}$ and $s_{2}$ defined in [12] as:

$$
\gamma_{s_{1} s_{2}}(\nu, \tau)=\overline{S_{s_{1}}}(\nu, \tau) * * \overline{S_{s_{2}}}(\nu, \tau),
$$

which depends on the normalized spectrum of the signals $\overline{S_{s_{1,2}}}(\nu)$ and their central frequency. The GSSC for $Y_{d i_{r}}$ and $Y_{i_{d} r}$ is obtained from [12]. The GSSC for $Y_{n_{d} n_{r}}$ is calculated as in [12]:

$$
\gamma_{n_{d} n_{r}}(\nu, \tau)=\frac{1}{B \cdot T_{c}},
$$

where $B$ is the system bandwidth, and $T_{c}$ the coherent integration time.

The GSSC for $Y_{n_{d} r}, Y_{d n_{r}}, Y_{i_{d} n_{r}}$, and $Y_{n_{d} i_{r}}$ is derived from Fig. 8, which shows all possible cross-correlations between the separate components of two signals composed by a GNSS code (L5 or E5), a pair of DME pulses, and noise have been simulated. Figure 8 shows the squared auto-correlation of the code (L5 or E5) in blue, the squared auto-correlation of a DME signal in red, the squared cross-correlation of the code against the noise in magenta, the squared cross-correlation of the DME against the code in black, and the squared crosscorrelation of the DME signal against the noise in green. For the L5 case, all signals have $20 \mathrm{MHz}$ bandwidth, while for the E5 case they have $50 \mathrm{MHz}$. All signals have been generated at $400 \mathrm{MS} / \mathrm{s}$, and have unit energy. The DME signals have been centered at $1,176.45 \mathrm{MHz}$. The figures are obtained as the incoherent averaging of 100 realizations of noise, and 32 different PRN codes.

Figure 8 shows that the squared auto-correlation function of the DME signal has 3 lobes, a main one and two side lobes 6 $\mathrm{dB}$ below. Each lobe lasts $12 \mu \mathrm{s}$, and are separated $12 \mu \mathrm{s}$. As

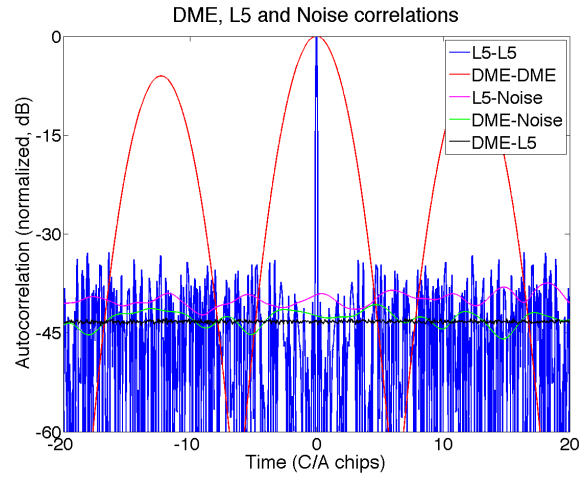

(a)

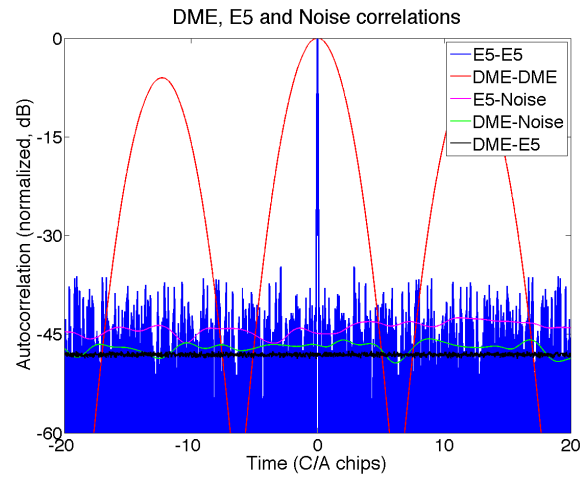

(b)

Fig. 8: (a) L5, DME and Noise auto and cross-correlations and (b) E5, DME and Noise auto and cross-correlations.

a consequence, and considering the high transmitted power, the correlation of GNSS signals might be easily masked. It can also be noticed that the DME-PRN, and DME-noise crosscorrelations increase the noise floor, which farther degrades the SNR. In both cases, the DME-code, and the DME-noise terms have a similar cross-energy than the code-noise term with unitary energy signals, but considering the high transmitted power of the DME system, the total impact is higher; which degrades the SNR even in navigation receivers. Figure 9 shows the impact of having a single pair of DME pulses inside the coherent integration time. To do so, real data was captured with a GNSS L1/E1 and L5/E5 dual-band antenna [18], and a Software Defined Radio [19]. The data shows the presence of DME signals at the channel corresponding to the Barcelona airport (12 km far from the experiment location, out of the lineof-sight). One millisecond of data without DME pulses, and another one with a single pair of DME pulses were selected. The presence of a single pulse in the correlation window implied a SNR degradation of $5 \mathrm{~dB}$.

\section{B. Traffic properties}

Figure 10 shows a sample of the recorded data. The analysis of this data shows that the time between pulses follows an exponential distribution, which means that the probability of having $N$ pulses in $T$ seconds with $\lambda$ mean arrivals per second follows a Poisson distribution. In GNSS-R, the data 


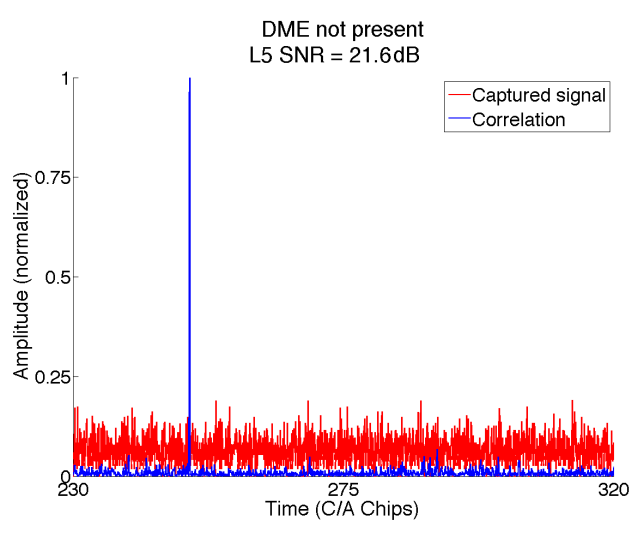

(a)

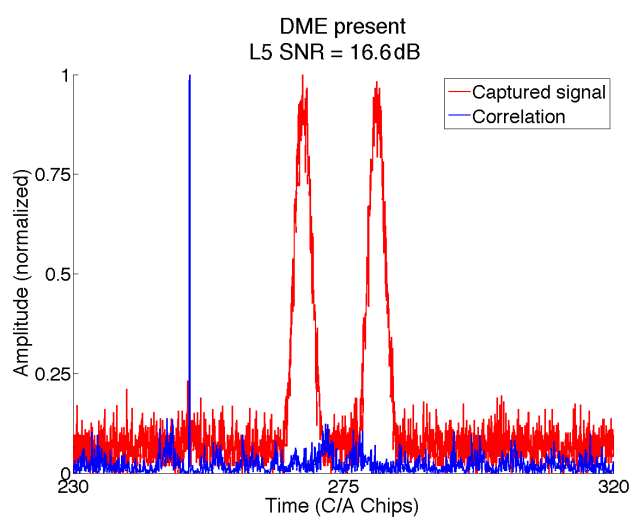

(b)

Fig. 9: SNR degradation in the presence of a single DME pulse in the coherent integration time.

is correlated during $T_{c}=1 \mathrm{~ms}$ and then incoherently averaged in (1). The resulting Probability Density Function (PDF) of the time between arrivals is computed as the modulo $T_{c}$ of an exponential random variable, and it is a uniform random variable.

From Fig. 10a, an average of 935 DME pulses per second is estimated, which represents around 35 planes simultaneously. This is consistent with the fact that not even the busiest airports reach the DME stations capabilities (100 planes simultaneously). As a consequence of being a Poisson random variable, the traffic arriving from $K$ transponders with $\lambda_{1}, \lambda_{2}, \ldots, \lambda_{K}$ arrival rates, also follows a Poisson distribution with $\lambda=\sum \lambda_{k}$.

\section{APPLICATION TO GNSS-R FROM A LEO ORBIT}

In order to estimate the impact of these signals in upcoming iGNSS-R instruments in LEO orbit, the Signal to Interferenceplus-Noise Ratio (SINR) will be computed. The SNIR is useful to estimate the impact for long incoherent averaging times. In this situation, the pulses are smoothed while averaging, resulting in an increase of the noise floor. First, the visibility and received power from all possible DME stations to an hypothetical receiver at $400 \mathrm{~km}$ height on-board the ISS [13] must be estimated. It will be computed for latitudes between $\pm 52^{\circ}$, the maximum ones that reach the ISS.

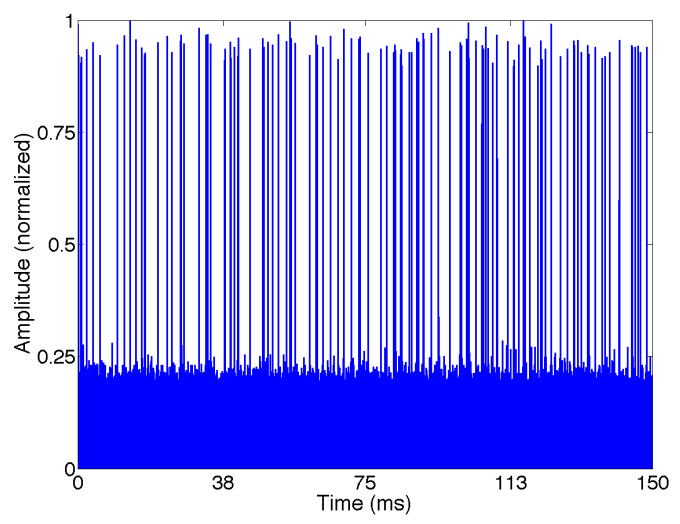

(a)

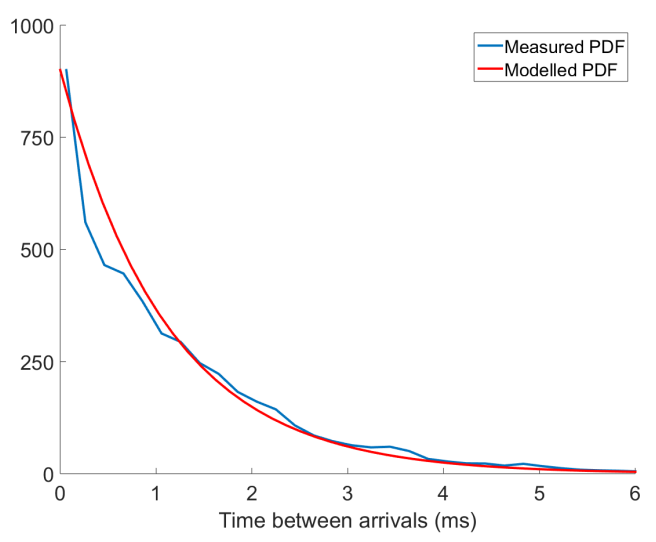

(b)

Fig. 10: (a) Sample of the captured data from the Barcelona Airport, and (b) PDF of the measured time between arrivals and theoretical exponential PDF with $\lambda=935$.

First, the visibility of the receiver towards the stations, the arrival angle of the interference, and its expected Equivalent Isotropic Radiated Power (EIRP) has been computed. To do so, the Earth has been modeled using the WGS-84 ellipsoid with standard atmospheric refraction [20]. DME stations out of the Line-of-Sight (LoS) affect due to diffraction effects [21].

It has been considered free space transmission. The transmitted power for all DME stations in [14] has been considered $1 \mathrm{~kW}$ for DME stations and $3.5 \mathrm{~kW}$ for TACAN stations [22]. A commercial radiation pattern has been considered for the DME stations [23]. The radiation pattern is omnidirectional in azimuth with a directivity of $9.5 \mathrm{dBi}$, has its main beam pointing at $4^{\circ}$ elevation, and transmitting at vertical polarization.

Then, the expected received power at the system has been calculated. To do so, the arrays proposed for the GNSS reflectometer of the GEROS-ISS experiment [13] are used (see Fig. 11) for the simulation. These arrays have 31 elements with a separation between elements of $0.93 \lambda_{L 1}$ and a directivity of $22 \mathrm{dBi}$ [24]. The radiation pattern of each radiating element has been approximated as:

$$
D(\theta)=D_{\max }-12 \cdot\left(\frac{\theta}{\theta_{-3 d B}}\right)^{2} \quad[d B]
$$


where $\theta$ is the antenna off-boresight angle, $D_{\max }$ is the maximum directivity $(8.4 \mathrm{dBi})$, and $\theta_{-3 d B}$ is the antenna beamwidth at $-3 \mathrm{~dB}\left(75^{\circ}\right)$. This model is a good approximation for the frontside of the antenna only. Since the reflectometer uses uses circular polarization, there are $3 \mathrm{~dB}$ of polarization losses in receiving the vertical polarized DME signals.

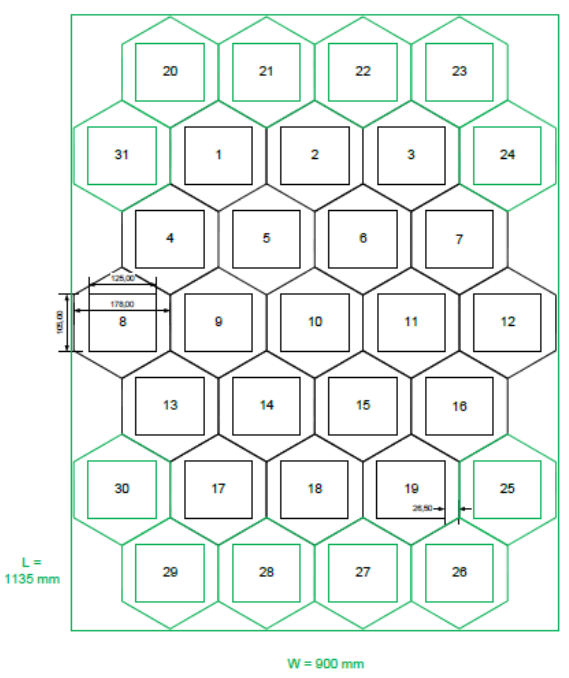

Fig. 11: Array used for the interference power simulation [24].

In order to compute the expected received power before correlation in any possible position of the receiver (latitudes in the range of $\pm 52^{\circ}$, and longitudes in the range of $\pm 180^{\circ}$, both in steps of $1^{\circ}$ ), the arrays have been pointed to elevations from $90^{\circ}$ to $50^{\circ}$ in steps of $2.5^{\circ}$, and all azimuths in steps of $5^{\circ}$. Then, the coefficients in (3) and (4) have been estimated.

The "delayed cross-correlation" has been considered. The DME and GNSS correlation peaks are assumed to be uniformly distributed in the delay axis. DME signals from different stations are received with uniformly distributed Doppler frequency in the $\pm 28 \mathrm{kHz}$ range, while GNSS peaks are uniformly distributed with random Doppler frequency in the $\pm 40 \mathrm{kHz}$ range. The Doppler frequency of the direct and the reflected signals from the same DME station are the same, and they change at a rate smaller than $1 \mathrm{~Hz} / \mathrm{ms}$ [17]. In these conditions, the $\Upsilon(\nu)$ function from (6) has been approximated empirically by:

$$
\Upsilon(\nu)=-2-\frac{|\nu|}{40 k H z} \quad[d B] .
$$

In order to estimate the average number of pulses per ms in (6) and (7), it has been considered that the air traffic follows the density of DME stations. Areas with higher traffic, such as North America, Europe, and the East coast of China, have more density of DME transponders. As consequence, the traffic has been distributed uniformly between all DME stations, and has been quantified in 13,000 aircraft flying simultaneously ${ }^{3}$. This means an average of 3.17 planes per DME

\footnotetext{
${ }^{3}$ Several websites provide in real time the position of all the planes in the world that use the Automatic Dependent Surveillance-Broadcast (ADS-B) system. However, not all commercial flights use this system. These websites show a peak of 15,000 planes during daylight in Europe and United States.
}

station. This consideration is not realistic for on-route areas between continents, such as Azores, Hawaii islands, Canary Islands, Japan, South-East Asia, Qatar, or the Caribbean Sea. As mentioned above, aircraft send an average of 27 PPS, but DME transceivers implement a $60 \mu \mathrm{s}$ dead time after transmitting a pair of pulses to avoid retransmitting echos which causes the loss of some pulses [25]. Consequently, only an average of 26 PPS are retransmitted. In summary, each transponder sends an average of 82.52 PPS, with a mean time between arrivals of $12.12 \mathrm{~ms}$.

In order to compute the useful term $Y_{d r}$ from (3) and (4), it has been considered that the expected power for the GPS direct signal is $-157 \mathrm{dBW}$ plus the antenna gain $(22 \mathrm{~dB})$, and the expected power for GPS reflected signal is $25 \mathrm{~dB}$ below [11]. The expected power for the Galileo direct signal is -155 $\mathrm{dBW}$ plus the antenna gain $(22 \mathrm{~dB})$, and similarly the Galileo reflected signal is expected to be $25 \mathrm{~dB}$ below. To determine the noise power, the system has a bandwidth of $20.46 \mathrm{MHz}$ for $\mathrm{L} 5$, and $51.15 \mathrm{MHz}$ for E5.

Finally, the SINR is computed for all pointing directions. For cGNSS-R it is computed as:

$$
\operatorname{SINR}_{\mathrm{c}}=\frac{\left\langle\left|Y_{c r}\right|^{2}\right\rangle}{\left\langle\left|Y_{c i_{r}}+Y_{c n_{r}}\right|^{2}\right\rangle},
$$

and for iGNSS-R as:

SINR $_{\mathrm{i}}$

$=\frac{\left\langle\left|Y_{d r}\right|^{2}\right\rangle}{\left\langle\left|Y_{d i_{r}}+Y_{d n_{r}}+Y_{i_{d} r}+Y_{i_{d} i_{r}}+Y_{i_{d} n_{r}}+Y_{n_{d} r}+Y_{n_{d} i_{r}}+Y_{n_{d} n_{r}}\right|^{2}\right\rangle}$.

In absence of interferences, the SINR becomes the SNR. For the assumed power levels, the top-boundaries of the SNR in cGNSS-R is $14 \mathrm{~dB}$ for L5, and $16 \mathrm{~dB}$ for E5. In the case of iGNSS-R, the boundaries are $8.4 \mathrm{~dB}$ for L5, and $8.9 \mathrm{~dB}$ for E5.

Besides, the degradation of the SINR caused by the interference terms for the conventional technique is computed as:

$$
\Delta_{\operatorname{SINR}_{\mathrm{c}}}=\frac{\left\langle\left|Y_{c i}+Y_{c n}\right|^{2}\right\rangle}{\left\langle\left|Y_{c n}\right|^{2}\right\rangle}
$$

and for the interferometric one as:

$\Delta_{\mathrm{SINR}_{\mathrm{i}}}$

$=\frac{\left\langle\left|Y_{d i_{r}}+Y_{i_{d} r}+Y_{i_{d} i_{r}}+Y_{i_{d} n_{r}}+Y_{n_{d} i_{r}}+Y_{d n_{r}}+Y_{n_{d} r}+Y_{n_{d} n_{r}}\right|^{2}\right\rangle}{\left\langle\left|Y_{d n_{r}}+Y_{n_{d} r}+Y_{n_{d} n_{r}}\right|^{2}\right\rangle}$.

Last, to determine which are the most relevant terms in the degradation, each interfering term is compared to the total contribution from RFI as:

$$
\Delta_{I}=\frac{\left\langle\left|Y_{d i_{r}}+Y_{i_{d} r}+Y_{i_{d} i_{r}}+Y_{i_{d} n_{r}}+Y_{n_{d} i_{r}}\right|^{2}\right\rangle}{\left\langle\left|Y_{s_{1} s_{2}}\right|^{2}\right\rangle},
$$

where $Y_{s_{1} s_{2}}$ is the term to be studied. As higher and closer to 0 , the more relevance will have the term. 


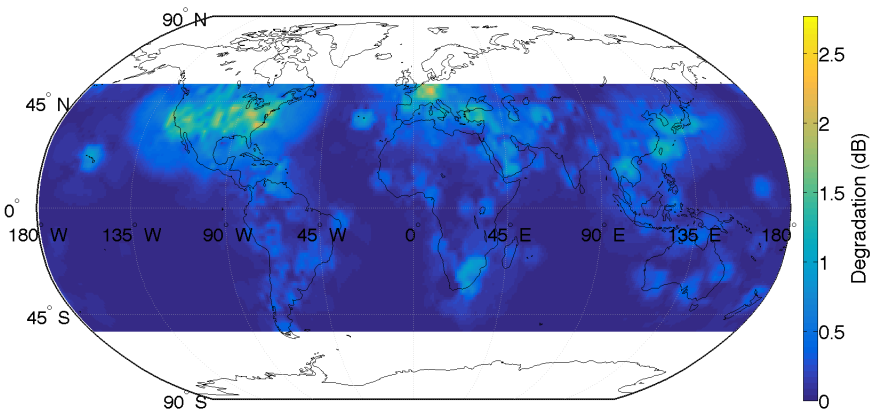

(a)

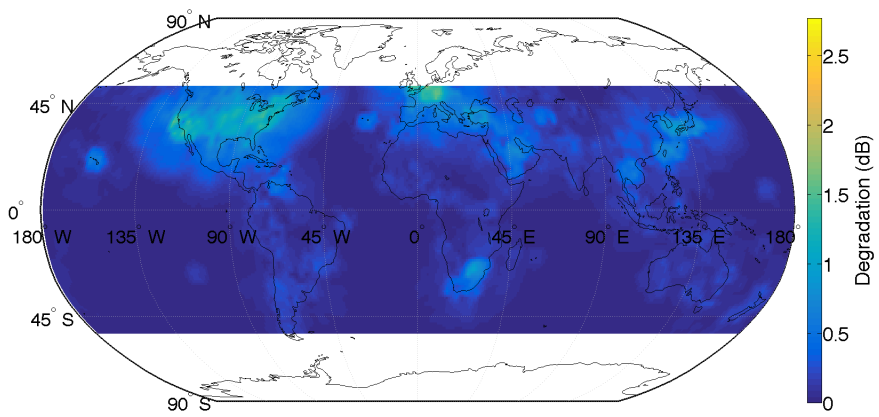

(b)

Fig. 12: Maximum expected SINR degradation for cGNSS-R in (a) L5 (maximum: $2.45 \mathrm{~dB}$ ), and (b) E5 (maximum: 1.7 $\mathrm{dB})$.

\section{DiscusSiON OF RESUlTS}

Figures $12 \mathrm{a}$ and $12 \mathrm{~b}$ show the maximum expected degradation in conventional GNSS-R for L5 and E5, respectively. Figures $13 \mathrm{a}$ and $13 \mathrm{~b}$ show the maximum expected degradation in interferometric GNSS-R for L5 and E5, respectively.

As expected, the highest degradation happens over the most populated areas in terms of DME stations: North America, Europe and the East coast of China. In the case of North America, the degradation could affect even far from the coast. The maximum degradation in cGNSS-R is $2.45 \mathrm{~dB}$ for L5 $(\mathrm{SNR}=14 \mathrm{~dB})$, and $1.7 \mathrm{~dB}$ for E5 $(\mathrm{SNR}=16 \mathrm{~dB})$. For iGNSS-R, the maximum degradation is $2.77 \mathrm{~dB}$ for L5 (SNR $=8.4 \mathrm{~dB})$, and $1.26 \mathrm{~dB}$ for E5 $(\mathrm{SNR}=8.9 \mathrm{~dB})$. These values can be used to study the impact on ocean altimetry using the equation (12) from [11]:

$$
\begin{aligned}
\sigma_{h}= & \frac{c \overline{P_{Z, S}}}{2 \sin \theta_{\text {elev }, S P} \overline{P_{Z, S}}} \cdot \frac{1}{\sqrt{N_{\text {inc }}}} \\
& \cdot \sqrt{\left(1+\frac{1}{\mathrm{SNR}}\right)^{2}+\left(\frac{1}{\mathrm{SNR}}\right)^{2}}
\end{aligned}
$$

where $c$ is the speed of light, $\overline{P_{Z, S}}$ is the total received power waveform, ${\overline{P_{Z, S}}}^{\prime}$ is the first derivative of the former, $\theta_{\text {elev }, S P}$ is the local elevation angle at the specular point, and $N_{i n c}$ is the number of incoherent averages. The relative degradation

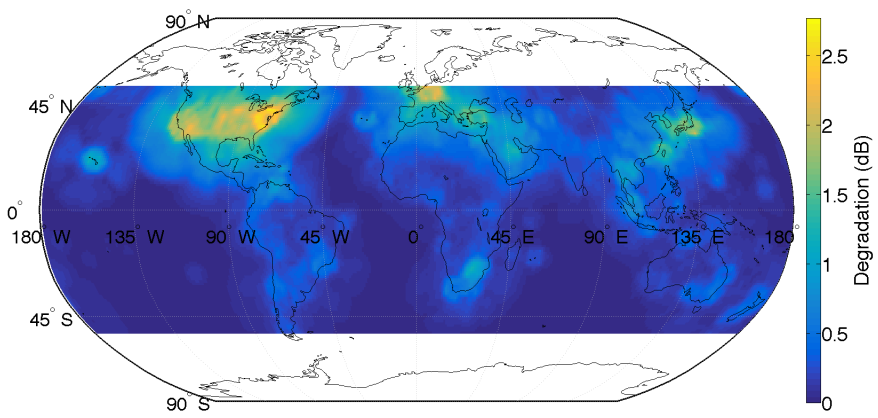

(a)

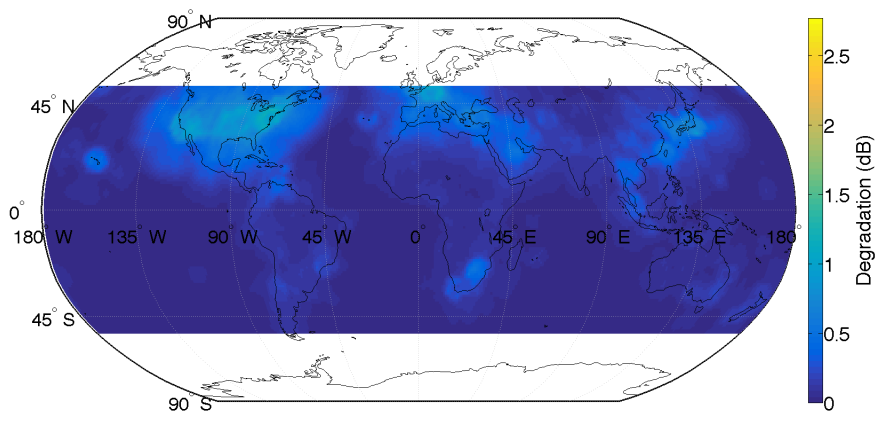

(b)

Fig. 13: Maximum expected SINR degradation for iGNSS-R in (a) L5 (maximum: $2.77 \mathrm{~dB}$ ), and (b) E5 (maximum: 1.26 $\mathrm{dB})$.

of the height precision caused by a degradation of the SINR can be computed as:

$$
\Delta \sigma_{h}=\frac{\sigma_{h}(\mathrm{SINR})}{\sigma_{h}(\mathrm{SNR})}
$$

Figure 14 shows the the Cumulative Distribution Function (CDF) of the relative degradation for the worst pointing case. It can be seen that the conventional technique is more robust against interferences than the conventional one, and L5 is more sensitive to RFI than E5. Despite this, even in the worst pointing case, the precision height degradation will be lower than $4 \%$ with a probability higher than $90 \%$. However, these results are extremely dependent on the antenna directivity which drives the SNR. Therefore, a lower directivity antenna will be more prone to suffer from RFI than a highly one.

Finally, table II presents which are the most dominant terms in the degradation for L5 and E5 for worst pointing cases. The most powerful terms result from the interference coming from the down-looking antenna, where it is received with more power. In both cases, the largest contribution is due to the noise in the up-looking antenna and the interference in the down-looking one.

\section{CONCLUSIONS AND FUTURE WORK}

This work has studied the DME/TACAN signals and how do they affect conventional and interferometric GNSS-R. Then, a methodology to simulate scenarios to study the potential degradation of a L5/E5 reflectometer has been presented, and the geographic areas more prone to disturb the system 


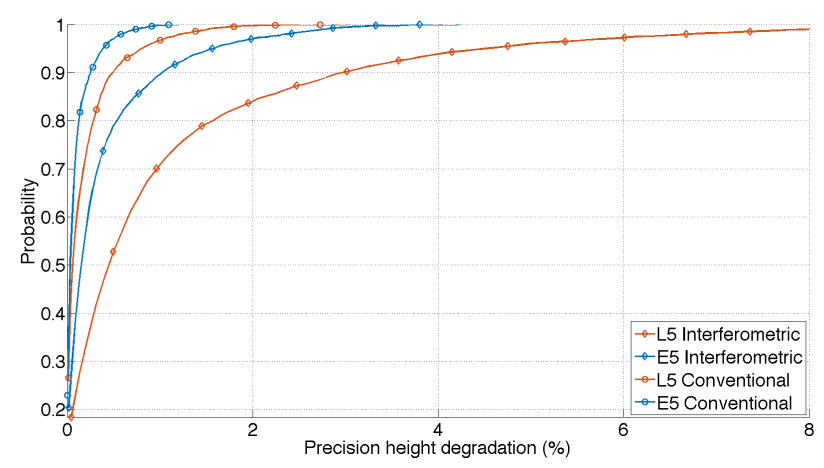

Fig. 14: CDF of the relative degradation of the height precision in sea altimetry in the worst pointing case.

TABLE II: Mean degradation in iGNSS-R caused by each interference cross-correlation term in L5 and E5, in the worst and best pointing cases.

\begin{tabular}{|r|r|r|r|r|r|}
\hline & $Y_{d i_{r}}$ & $Y_{i_{d} r}$ & $Y_{i_{d} i_{r}}$ & $Y_{i_{d} n_{r}}$ & $Y_{n_{d} i_{r}}$ \\
\hline L5 & $-7.6 \mathrm{~dB}$ & $-55.9 \mathrm{~dB}$ & $-34.4 \mathrm{~dB}$ & $-23.6 \mathrm{~dB}$ & $-0.85 \mathrm{~dB}$ \\
\hline E5 & $-6.07 \mathrm{~dB}$ & $-54.2 \mathrm{~dB}$ & $-29.5 \mathrm{~dB}$ & $-24.1 \mathrm{~dB}$ & $-1.3 \mathrm{~dB}$ \\
\hline
\end{tabular}

analyzed. In particular, a GEROS-ISS like GNSS-R instrument has been simulated at LEO. The results show that for a 22 $\mathrm{dBi}$ directivity antenna a degradation of the height precision smaller than $4 \%$ can be expected $90 \%$ of the time . Even with such a small degradation, an iGNSS-R instrument should have a RFI mitigation system included such a pulse blanking one [24]. This work has studied one particular kind of signal that shares the L5/E5 band, but other kind of interferences could severely degrade the system performance, such as out-of-band interferences or jamming as TechDemoSAT-1 has experienced at L1.

The dominant degradation term of the cross-correlation in iGNSS-R is the resulting of cross-correlating the captured DME/TACAN signals in the down-looking antenna against the noise in the up-looking antenna (iGNSS-R), and not the crosscorrelation of the interferences in both antennas as it could be expected a priori.

The impact on airborne instruments is not he object of this study, but it can be anticipated that it is even worst. First, because DME/TACAN signals are received much stronger. Second, because in the spaceborne case, the "delayed crosscorrelation" spreads the energy of the DME-DME cross-term along the delay axis, while in ground-based and airborne systems, all the energy is concentrated at the origin of the DDM plane. Besides, as in the spaceborne case, the crosscorrelation of interferences and noise dramatically increases the noise floor.

\section{ACKNOWLEDGMENTS}

Thanks to F. Moreno García, and J. Gelabert, from ENAIRE (the company designated by the Spanish government to provide air traffic services in the on-route and approximation phases), for their help the authors providing information about the DME system and the Spanish airports.

\section{REFERENCES}

[1] C. Hall and R. Cordey, "Multistatic Scatterometry," in International Geoscience and Remote Sensing Symposium, 'Remote Sensing: Moving Toward the 21st Century'., vol. 1. IEEE, 1988, pp. 561-562.

[2] M. Martin-Neira, "A Passive Reflectometry and Interferometry System (PARIS): Application to Ocean Altimetry," ESA Journal, vol. 17, pp. 331-355, 1993.

[3] V. Zavorotny and A. Voronovich, "Scattering of GPS signals from the ocean with wind remote sensing application," IEEE Transactions on Geoscience and Remote Sensing, vol. 38, no. 2, pp. 951-964, mar 2000.

[4] S. Katzberg and J. Garrison, "Utilizing GPS To Determine Ionospheric Delay Over the Ocean," NASA Technical Memorandum 4750, 1996.

[5] OACI and ICAO, "Annex 10: Aeronautical Telecommunications," ICAO - OACI, Tech. Rep. November, 1996.

[6] M. De Angelis, R. Fantacci, S. Menci, and C. Rinaldi, "Analysis of air traffic control systems interference impact on galileo aeronautics receivers," in IEEE International Radar Conference, 2005. IEEE, 2005, pp. 585-595.

[7] F. Dovis, L. Musumeci, and J. Samson, "Performance assessment of pulse blanking mitigation in presence of multiple Distance Measuring Equipment/Tactical Air Navigation interference on Global Navigation Satellite Systems signals," IET Radar, Sonar \& Navigation, vol. 8, no. 6, pp. 647-657, jul 2014.

[8] E. D. Kaplan and C. J. Hegarty, Understanding GPS. Principles and Applications, 2nd ed., Artech House, Ed., 2005.

[9] J. W. Betz, "Effect of partial-band interference on receiver estimation of C/N0: Theory," The MITRE Corporation, Tech. Rep., 2001.

[10] B. Roturier, "Report on DME interference on GPS/L5," Eurocontrol, Tech. Rep., 2001, Last visit: 2015-10-25. [Online]. Available: https: //www.eurocontrol.int/sites/default/files/field_tabs/content/documents/ communications/071999-dgac-report-dme-interference-on-gps-15.pdf

[11] M. Martín-Neira, S. D'Addio, C. Buck, N. Floury, and R. PrietoCerdeira, "The PARIS ocean altimeter in-Orbit demonstrator," IEEE Transactions on Geoscience and Remote Sensing, vol. 49, no. 6 PART 2, pp. 2209-2237, 2011.

[12] J. Querol, A. Alonso-Arroyo, R. Onrubia, D. Pascual, H. Park, and A. Camps, "SNR degradation in GNSS-R measurements under the effects of Radio-Frequency Interference," IEEE Journal of Selected Topics in Applied Earth Observations and Remote Sensing, submitted for publication, 2015.

[13] J. Wickert, O. B. Andersen, G. Beyerle, B. Chapron, E. Cardellach, S. D'Addio, C. Foerste, C. Gommenginger, T. Gruber, A. Helm, M. Hess, P. Hoeg, A. Jaeggi, N. Jakowski, M. Kern, T. Lee, M. Martin-Neira, O. Montenbruck, N. Pierdicca, A. Rius, M. Rothacher, C. Shum, and C. Zuffada, "GEROS-ISS: Innovative GNSS reflectometry/occultation payload onboard the International Space Station for the Global Geodetic Observing System," in Proc. ARSI-KEO workshop, Nov. 4-7, ESTEC, Noordwijk, 2014.

[14] Megginson Technologies Ltd., "OurAirports," Last visit: 2015-10-25. [Online]. Available: http://ourairports.com/data/

[15] T. Elfouhaily, D. Thompson, and L. Linstrom, "Delay-Doppler analysis of bistatically reflected signals from the ocean surface: theory and application," IEEE Transactions on Geoscience and Remote Sensing, vol. 40, no. 3, pp. 560-573, 2002.

[16] A. Camps, H. Park, E. Valencia i Domenech, D. Pascual, F. Martin, A. Rius, S. Ribo, J. Benito, A. Andres-Beivide, P. Saameno, G. Staton, M. Martin-Neira, S. DAddio, and P. Willemsen, "Optimization and Performance Analysis of Interferometric GNSS-R Altimeters: Application to the PARIS IoD Mission," IEEE Journal of Selected Topics in Applied Earth Observations and Remote Sensing, vol. 7, no. 5, pp. 1436-1451, may 2014.

[17] H. Park, D. Pascual, A. Camps, F. Martin, A. Alonso-Arroyo, and H. Carreno-Luengo, "Analysis of Spaceborne GNSS-R Delay-Doppler Tracking," IEEE Journal of Selected Topics in Applied Earth Observations and Remote Sensing, vol. 7, no. 5, pp. 1481-1492, may 2014.

[18] R. Onrubia and A. Camps, "Antena Multibanda Tipo Parche con Sistema de Alimentación Cruzada," Spain Patent ES2 540161 A1, October, 2015.

[19] Ettus Research. (2015) Ettus Research. Last visit: 2015-10-25. [Online]. Available: http://www.ettus.com

[20] ITU-R, "P.452-15: Prediction procedure for the evaluation of interference between stations on the surface of the Earth at frequencies above about 0.1 GHz," Tech. Rep., Last visit: 2015-10-25. [Online]. Available: http://www.itu.int/rec/R-REC-P.452-15-201309-I/en

[21] — "P.526-13: Propagation by diffraction," Tech. Rep., Last visit: 2015-10-25. [Online]. Available: http://www.itu.int/rec/R-REC-P. 526-13-201311-I/es 
[22] F. Moreno, ENAIRE, Private communication, February 2016.

[23] dB Systems Inc., "Omni-Directional dBs 5100A DME Antenna." [Online]. Available: http://www.dbsant.com/5100A.php

[24] J. Benito, ESA, AIRBUS, IEEC/UPC, MIER, RUAG, OHB, and DEIMOS, "GEROS-ISS Performance and Error Budget Report: Part 1/3 Assessment of RFI Effects in GNSS-R GEROS-ISS-PhA-IEEC-UPCTN-D26-1/3," Tech. Rep., 2015.

[25] B. Forssell, Radionavigation Systems, Prentice Hall, Ed., 1991, pp. 166175 .

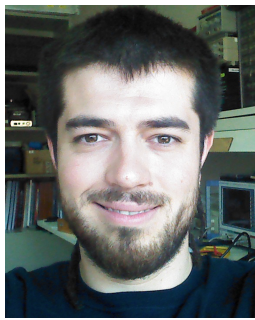

Raul Onrubia (S'10)was born in Barcelona, Spain. $\mathrm{He}$ received the B.Sc. degree $(\mathrm{BSc}+5)$ in telecommunications engineering the M.Sc. degree $(\mathrm{Msc}+2)$ in research on information and communication technologies from the Universitat Politécnica de Catalunya, Barcelona, Spain in 2012 and 2014, respectively. He is currently working toward the $\mathrm{PhD}$. degree in GNSS-Reflectometry with the Passive Remote Sensing Group, Signal Theory and Communications Department, Universitat Politècnica de Catalunya-BarcelonaTech, Barcelona, Spain. His current work is the development of RF hardware, and the study of interferences and mitigation techniques.

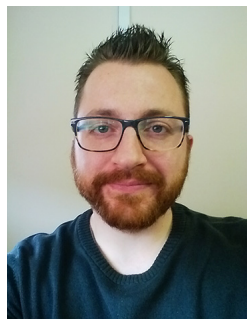

Jorge Querol (S'13) was born in Castelló, Spain, in 1987. He received the M.Sc. degree in electronics engineering, M.Sc. degree in telecommunication engineering and M.Sc. degree in photonics from the UPC-BarcelonaTech in 2011, 2012 and 2013, respectively. Currently, he is a Ph.D. candidate working as graduate research assistant at the Remote Sensing Laboratory (RSLab) at UPCBarcelonaTech, Barcelona, Spain. His research deals with the development of real-time signal processing systems able to detect and mitigate the effects of Radio-Frequency Interference (RFI) signals and jamming in Global Navigation Satellite Systems (GNSS) and Passive Remote Sensing (PRS) applications, particularly, those ones working in L-band such as MicroWave (MW) radiometry and GNSS-Reflectometry (GNSS-R).

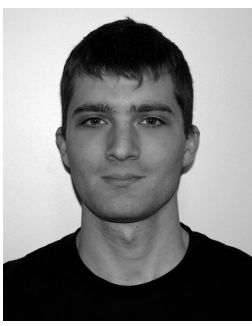

Daniel Pascual (S'11) was born in Barcelona, Spain, in 1985. He received the $\mathrm{BSc}+5$ degree in telecommunications engineering specialized in communications in 2011, and the MSc+2 degree in Research on Information and Communication Technologies in 2014, both from the Universitat Politècnica de Catalunya (UPC), Barcelona, Spain. In 2011 he joined the Passive Remote Sensing Group from UPC where he is currently working toward the $\mathrm{PhD}$ degree in GNSS-Reflectometry focused in ocean altimetry.

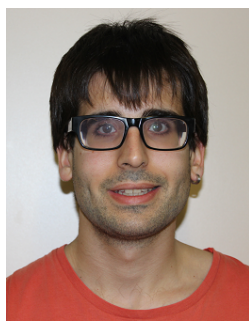

Alberto Alonso Arroyo (S'11) was born in Barcelona, Spain. He received the M.S. degree in telecommunications engineering in $2011(\mathrm{BSc}+5)$ and the M.S. in Research on Information and Communication Technologies in $2012(\mathrm{MSc}+2)$, both from the Universitat Politècnica de CatalunyaBarcelonaTech. He is working toward the Ph.D. degree in GNSS-Reflectometry, with the Passive Remote Sensing Group, Department of Signal Theory and Communications, at the Universitat Politècnica de Catalunya-BarcelonaTech. Currently, he is at $\mathrm{Na}-$ tional Oceanic and Atmospheric Administration (NOAA) as an invited visiting resear cher thanks to a Fulbright grant.

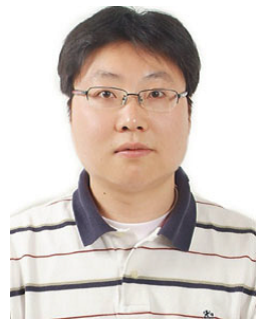

Hyuk Park (S'05-AM'09-M'12-SM'15) was born in South Korea. He received the B.S. degree in mechanical engineering from the Korea Advanced Institute of Science and Technology (KAIST) in 2001, and received the M.S. and Ph.D. degree in information and mechatronics from the Gwangju Institute of Science and Technology (GIST), Korea, in 2003 and 2009, respectively. His main research interest is in the area of remote sensing, especially passive microwave remote sensing, including system design, modeling and simulation, and image processing. In 2009, he joined the remote sensing group of the Polytechnic University of Catalonia (UPC), Barcelona, as a postdoctoral researcher. He is a grant holder of NRF funded by Korean government in 2011. From 2012, he has been working as a research associate with Grant of Juan de la Cierva funded by Spanish Ministry of Economy and Competitiveness. Currently, he is working with the passive remote sensing group in the UPC for satellite remote sensing for microwave radiometry and GNSS-R. 


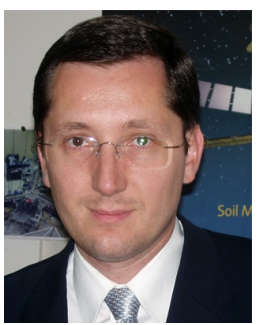

Adriano Camps (S'91-A'97-M'00-SM'03-F'11) was born in Barcelona, Spain, in 1969. He received the degree in Telecommunications Engineering and the Ph.D. degree in telecommunications engineering from the Universitat Politècnica de Catalunya (UPC), Barcelona, Spain, in 1992 and 1996, respectively. From 1991 to 1992 , he was with the ENS des Télécommunications de Bretagne, France, with an Erasmus Fellowship. Since 1993, he has been with the Electromagnetics and Photonics Engineering Group, Department of Signal Theory and Communications, UPC, where he was an Assistant Professor first, an Associate Professor in 1997, and a Full Professor since 2007. In 1999, he was on sabbatical leave at the Microwave Remote Sensing Laboratory, University of Massachusetts, Amherst, MA, USA. Since 1993, he has been deeply involved in the European Space Agency SMOS Earth Explorer Mission, from the instrument and algorithmic points of view, performing field experiments and more recently studying the use of Global Navigation Satellite Systems Reflectometry (GNSSR) techniques to perform the sea state correction needed to retrieve salinity from radiometric observations. His research interests are focused in microwave remote sensing, with special emphasis in microwave radiometry by aperture synthesis techniques, remote sensing using signals of opportunity (GNSSR), and the development of nanosatellites to test innovative remote sensing techniques. Dr. Camps served as Chair of MicroCal (uCal) 2001, Technical Program Committee Co-chair of IEEE International Geosciences and Remote Sensing Symposium (IGARSS) 2007, and Co-chair of GNSS-R 2010. He was an Associate Editor of Radio Science, and he is an Associate Editor of the IEEE Transactions on Geoscience and Remote Sensing and the President-Founder of the IEEE Geoscience and Remote Sensing Chapter in Spain. He was a recipient of the Second National Award of University Studies, in 1993; the INDRA Award of the Spanish Association of Telecommunication Engineers to the Best Ph.D. in Remote Sensing, in 1997; the Extraordinary Ph.D. Award at the UPC, in 1999; the Research Distinction of the Generalitat de Catalunya, for contributions to microwave passive remote sensing, in 2002; the European Young Investigator Award in 2004; and the Institució Catalana de Recerca i Estudis Avançats (ICREA) Academia Award in 2009 and 2014. Moreover, as a member of the Microwave Radiometry Group, UPC, he received the 1st Duran Farell and the Ciutat de Barcelona Awards for Technology Transfer, in 2000 and 2001, respectively, and the "Salvà i Campillo" Award of the Professional Association of Telecommunication Engineers of Catalonia for the most innovative research project for Microwave Imaging Radiometer by Aperture Synthesis/Soil Moisture and Ocean Salinity mission (MIRAS/SMOS)-related activities, in 2004, and the 7th Duran Farell Award for Technological Research, for the work on GNSS-R instrumentation and applications, in 2010. 